\title{
Actualización \\ Constipación en el paciente adulto. Manejo en el consultorio de atención primaria
}

La constipación es un motivo de consulta frecuente en el consultorio de atención primaria ya que provoca una sensación de disminución de la calidad de vida. ${ }^{1}$ Afecta a pacientes de todas las edades y es más frecuente en pacientes ancianos $(26 \%$ hombres y $34 \%$ mujeres)

Si bien existen numerosos métodos diagnósticos y terapéuticos la evaluación inicial del paciente debe centrarse en la historia clínica y el examen físico, siendo ésto suficiente en la mayoría de los casos para iniciar un tratamiento empírico. En los casos refractarios o cuando se sospecha una patología subyacente seria, estaría indicado realizar estudios diagnósticos.

\section{Definición}

La constipación puede ser definida por una disminución en la frecuencia del ritmo evacuatorio, por el pasaje de heces duras o por mayor esfuerzo defecatorio. Si bien existe una gran variación en la frecuencia evacuatoria en la población adulta (de tres veces por día a dos veces por semana), cualquier disminución en el ritmo evacuatorio habitual suficiente para causar disconfort, debe ser considerada constipación y requiere evaluación por parte del médico.

Otra forma de definirla es según el mecanismo que la produce: funcional versus trastornos de la evacuación rectal. Segun un reporte de un trabajo internacional 2 en el manejo de la constipación la misma puede definirse de la siguiente forma:

- Funcional (dos o más de los hallazgos siguientes el $25 \%$ de tiempo por al menos 12 meses, sin haber consumido laxantes): esfuerzo defecatorio, heces duras, sensación de evacuación incompleta o menos de dos evacuaciones semanales.

- Retraso en la evacuación rectal: bloqueo anal el $25 \%$ del tiempo o más y defecación prolongada o necesidad de desimpactación manual.

\section{Definición}

- Disminución en la frecuencia del ritmo evacuatorio

- Heces duras

- Mayor esfuerzo defecatorio

- Cualquier disminución del ritmo evacuatorio habitual + disconfort

\section{Causas}

Existen numerosos factores que pueden afectar la motilidad colónica, entre ellos una dieta con un inadecuado aporte de fibras es la causa más frecuente. Aunque la recomendación diaria de aporte en la dieta es de 25 grs., las personas con dietas con al menos 15 gramos de fibras por día tienen el doble de deposiciones semanales que los pacientes con dietas libres (dieta común en Occidente) 1 . Otras causas pueden ser clasificadas dentro de las siguientes categorías 3 :

- Síndrome del intestino irritable: Entre los pacientes con sindrome del intestino irritable la constipación puede ser el síntoma predominante en muchos de ellos. En estos casos el tratamiento más adecuado consiste en un incremento de fibra en la dieta y el manejo del hábito evacuatorio.

- Tránsito colónico lento: El tránsito colónico lento es la causa no obstructiva más frecuente de constipación en ancianos. El tránsito colónico normal es de 18-72 hs. (promedio 35). En los pacientes con tránsito colónico lento el tiempo es mayor de $72 \mathrm{hs}$. Se cree que esta entidad se debería a desórdenes del plexo mientérico y que seria parte de un trastorno motor gastrointestinal generalizado. La inercia colónica se considera como un subgrupo dentro del tránsito colónico lento, definida como la falta de respuesta del cólon a estimulantes como el bisacodylo. ${ }^{2}$

- Disfunción del piso pelviano (desordenes de la evacuación) Para una defecación normal se necesita que una serie de eventos ocurra de una manera coordinada: la relajación del músculo pubo-rectal y del esfínter anal externo, la apertura del ángulo ano-rectal y el aumento de la presión intraluminal con el esfuerzo.

Cuando el paciente presenta sensación de evacuación incompleta o gran esfuerzo defecatorio, orientaría a una disfunción del piso pelviano. Cuando la presión de reposo del canal anal es elevada o no se produce relajación del esfínter anal durante la defecación (o ambos) se dificulta el pasaje de las heces y se denomina anismo.

El síndrome pubo-rectal se refiere a la falla de relajación del músculo pubo-rectal o a una contracción paradójica con el esfuerzo, lo que provoca una obstrucción funcional a la salida de la materia fecal.

El síndrome del periné descendido se refiere a un excesivo descenso de los músculos del piso pelviano al disminuir el tono de los mismos como resultado de años de esfuerzo, partos vaginales múltiples y pobre dinámica defecatoria. El $50 \%$ de los pacientes con $\mathrm{s}$. del periné descendido experimentan incontinencia defecatoria.

Rectocele y enterocele: pueden causar obstrucción pero rara vez provocan constipación.

El rectocele ocurre más frecuentemente en mujeres por daño obstétrico y refleja la debilidad de la pared anterior de la vagina. Aunque puede ser asintomático, también puede causar sensación de evacuación incompleta.

El enterocele es la herniación del peritoneo en el cul de sac, lo que ocurre más frecuentemente en mujeres con cirugías pelvianas. La herniación puede llegar a la pared anterior del ano.

- Medicamentos: Si bien la lista de fármacos es larga, entre los medicamentos más comúnmente utilizados asociados a la constipación se pueden incluir: antiácidos (los que contienen aluminio y calcio) anticolinérgicos (antidepresivos y antihistamínicos), bloqueantes de los canales de calcio, hierro, levodopa, bario, bismuto, AINE, opiáceos, sucralfato, clonidina, etc.

- Enfermedades sistémicas: Entre las enfermedades sistémicas que más comúnmente se asocian a constipación podemos citar: diabetes mellitus, hipotiroidismo, hiperparatiroidismo (hipercalcemia), trastornos neurológicos (Parkinson, esclerosis múltiple, neuropatía autonómica), insuficiencia renal, etc.

- Anormalidades estructurales: Son aquellas que impiden el pasaje de materia fecal por un mecanismo obstructivo (ej. Cáncer) y aquellas que provocan dolor al defecar (fisura anal, hemorroides o enfermedad de Crohn recto-anal) 
- Congénitas: aunque es poco frecuente la enfermedad de Hirschprung (trastorno genético caracterizado por la ausencia de inervación de un segmento largo o corto del colon) se puede diagnosticar en pacientes de todas las edades. La misma se puede excluir mediante una manometría ano-rectal identificando la presencia del reflejo inhibitorio recto-anal.

\section{Causas de constipación}

- Bajo aporte dietario de fibras (la más común)

- Sme. del intestino irritable

- Tránsito colónico lento

- Disfunción del piso pelviano

- Inducida por medicamentos

- Enfermedades sistémicas

- Anormalidades estructurales

- Congénitas

\section{Evaluación inicial del paciente}

La historia clínica y el examen físico son útiles para orientar el diagnóstico de constipación. Es importante interrogar sobre la toma de medicamentos (los prescriptos por el médico y los que el paciente toma por su cuenta, incluída la medicación homeopática), la ingesta de fibras en la dieta y la presencia de síntomas asociados que orienten hacia la presencia de patología estructural o enfermedades sistémicas.

Según trabajos realizados 4 es útil determinar los síntomas principales que aquejan al paciente constipado dado que esto podría correlacionarse con los tres grupos principales propuestos para la constipación primaria o funcional: tránsito colónico lento, síndrome del intestino irritable y disfunción del piso pelviano. Si bien éstos trabajos pudieron demostrar la relación entre la presencia de síntomas tales como la disminución de la frecuencia evacuatoria intestinal (menos de tres veces por semana) y la falta de sensación de urgencia para evacuar el intestino con el tránsito colónico lento y la presencia de los criterios de Manning (aumento de la frecuencia de los movimientos intestinales con dolor, pérdida de los movimientos intestinales con dolor, distensión abdominal visible, sensación de evacuación incompleta, alivio del discomfort con los movimientos intestinales y pasaje de moco por el recto) para los pacientes con Sme. del intestino irritable, no pudieron demostrar de forma concluyente la misma correlación para los pacientes con disfunción del piso pelviano.

También son importantes los antecedentes patológicos del paciente (cirugías pelvianas previas) y los de su familia (p.ej. cáncer de colon en familiares de primer grado)

El examen físico debe considerar la presencia de enfermedades sistémicas (parkinson, hipotiroidismo, etc), y se debe realizar un examen detallado del área rectal para detectar masas, hemorroides externas, fisuras. El tacto rectal es útil para identificar hemorroides internas trombosadas, fisuras, masas rectales, estenosis, prolapso de la mucosa con el esfuerzo y defectos de la pared ano-rectal. El descenso perineal con el esfuerzo es determinado durante la inspección visual y durante el tacto rectal. El descenso normal es de 2 a $4 \mathrm{~cm}$. con el paciente en decúbito lateral izquierdo. Una falta de descenso orienta hacia una falta de relajación del piso pelviano y un descenso mayor de $4 \mathrm{~cm}$. hacia un $\mathrm{S}$. el periné descendido. La evaluación del tono del esfínter anal externo tiene poca correlación con la manometría rectal, salvo que sea claramente patológico. 5
Evaluación inicial del paciente

Historia clínica

Medicamentos

Ingesta de fibra Frecuencia evacuatoria <3/semana (orienta a Tránsito colónico lento)

Criterios de Manning (orientan a Sme. del intestino irritable)

Cirugías pelvianas previas

Antecedentes familiares de cáncer de colon

\section{Examen físico}

Signos de enfermedades sistémicas (Parkinson, hipotiroidismo, etc.)

Examen del área rectal

Descenso perineal con el esfuerzo

Tono del esfínter anal externo

\section{Manejo inicial del paciente}

El manejo inicial del paciente está determinado fundamentalmente por los hallazgos obtenidos durante la evaluación inicial del mismo y por la edad de comienzo de los síntomas.

El manejo empírico inicial consiste en aumentar la ingesta de fibra en la dieta y/o usar algún suplemento. ${ }^{6}$

El aporte de fibra diario debe ser de 25 a 30 grs. Para evitar la distensión abdominal y el meteorismo que son los efectos adversos más frecuentes, se debe incrementar la ingesta de fibra en forma lenta, por ej. 5 grs. día por semana hasta llegar a la dosis diaria recomendada. Aunque es común indicar el aumento de la ingesta de líquido y el ejercicio físico, esto no está claramente establecido. 7,8

En aquellos pacientes que no responden a las medidas anteriores o que no pueden cumplirlas, se puede comenzar utilizando laxantes de volumen; pero siempre debe tenerse en cuenta que los laxantes no constituyen el pilar del tratamiento. No se conoce en profundidad su mecanismo de acción y habitualmente se subestima su potencial toxicidad

Los laxantes más utilizados son los siguientes:

- Laxantes de volumen: ejercen su efecto fundamentalmente al absorber agua en la luz intestinal y aumentar el volumen fecal. Son laxantes efectivos y seguros y su efecto adverso más frecuente es el meteorismo, que desaparece con el uso continuo. Se han reportado casos de oclusión en pacientes con estenosis esofágicas e intestinales, y existe la posibilidad que interfieran con la absorción de Digital, Acenocumarol y Nitrofurantoina, por lo que debe tomarse lejos de las medicaciones. Ej. de los mismos son: Psyllium ( dosis 2 cucharaditas de te del polvo o $2 \mathrm{com}$ primidos por día) tener en cuenta que el efecto laxante comienza a partir de las 24 hs. y debe consumirse con abundante agua (250 ml. cada 2 cucharitas). Otros ej. de laxantes de volumen son: Policarbófilo Cálcico a una a dosis es de 2 a 8 tabletas por día y el Polietilenglicol en una dosis usual de 2 sobres por día.

- Laxantes estimulantes o irritativos: actúan aumentando la motilidad intestinal al alterar el transporte de electrolitos a través de la mucosa. Ej. de estos laxantes son la Fenolftaleina, el Bisacodyl, la Cascara sagrada y la Cassia Angustifolia. La fenoftaleína se asoció al sindrome de Stevens Johnson y el uso cróni- 
co de antraquinonas como la cáscara sagrada con daño del plexo mientérico. Pueden generar disturbios electrolíticos, diarrea facticia y nefritis. Pasan a la leche materna y pequeñas dosis pueden afectar al lactante. El uso de laxantes irritativos debe evitarse.

- Laxantes osmóticos: entre estos laxantes se encuentra el aceite de ricino (debe evitarse por el riesgo de disturbios electrolíticos) La lactulosa es un disacárido. semisintético que no se metaboliza por las enzimas intestinales. Se usa en pacientes con encefalopatía hepática y es efectivo en pacientes con constipación crónica. Es relativamente cara y requiere de 48 hs. para comenzar a actuar. Los efectos adversos más frecuentes son la distensión abdominal y el meteorismo. No debe usarse en dietas restringidas en galactosa y debe usarse con precaución en diabéticos. Otros agentes osmóticos son las sales de magnesio o sodio. Son útiles en preparaciones para estudios radiológicos Deben usarse con precaución en pacientes con función renal disminuida.

- Agentes emolientes (ablandadores): entre estos laxantes tenemos el docusato de sodio. Por lo general es bien tolerado. Ocasionalmente pueden producir calambres. Otros agentes como la vaselina pueden ablandar la materia fecal. Su uso crónico puede interferir con la absorción de vitaminas liposolubles.

Se debe resaltar que, excepto con los laxantes de volumen y los azúcares no absorbibles como la lactulosa y el sorbitol, el uso crónico de laxantes puede generar constipación a largo plazo.

Laxantes de volumen

Psyllium 2 cucharaditas de te del polvo o 2 comprimidos/día (250 ml. de agua cada 2 cucharitas)

Policarbófilo Cálcico 2 a 8 tabletas/día Polietilenglicol 2 sobres/día

Agentes emolientes (ablandadores)

Vaselina líquida $15-4.5 \mathrm{ml}$ diariamente al acostarse

Docusato de sodio $100-200 \mathrm{mg}$ diarios o 2 veces/día

Laxantes estimulantes
0 irritativos

Fenoftaleina

Bisacodyl

Cascara sagrada

Cassia Angustifolia

Laxantes osmóticos

Lactulosa 5-60 ml. día o 2 veces/día Sorbitol $70 \% 15-60 \mathrm{ml}$. o 2 veces por día

El círculo vicioso habitual es el siguiente: el paciente no evacua el intestino como cree que es saludable (falsas creencias acerca de la necesidad de evacuar todos los días) y recurre a un laxante estimulante. Luego de una deposición promovida por estos agentes suelen pasar 2 ó 3 días hasta lograr una deposición espontánea, lo cual acentúa la creencia por parte del paciente que está constipado y necesita más medicación y as sucesivamente. No hay ninguna ventaja en tener deposiciones frecuentes, excepto en pacientes con enfermedad hepática grave, ya que la constipación puede desencadenar encefalopatía.
Estudios complementarios: Evaluación selectiva del paciente Los estudios de laboratorio y/o de imágenes del cólon están indicados en aquellos pacientes que:

- no responden al tratamiento conservador y presentan constipación persistente

- en todos los pacientes que comienzan con los síntomas después de los 40 años

- cuando existe la sospecha clínica de patología orgánica subyacente

- y en aquellos pacientes en los que existe el antecedente de cáncer de colon en un familiar de primer grado.

Los estudios de laboratorio se solicitan en función de la evaluación inicial del paciente. Entre los estudios debe solicitarse: TSH ultrasensible, calcemia, glucemia, creatininemia, hemograma y determinación de sangre oculta en materia fecal

Entre los estudios por imágenes se incluyen colon por enema asociado a una rectosigmoideoscopía o una fibrocolonoscopía. En los casos en los que el paciente no responda a ninguna de las medidas anteriores y no se realice diagnóstico de patología subyacente o enfermedad sistémica, sería de utilidad realizar estudios fisiológicos. Esto permitiría determinar la causa de constipación en 3 de 4 pacientes, permitiendo de ésta forma adoptar las medidas terapéuticas que resulten más adecuadas. 9

Los tests más utilizados son los siguientes:

Medición del tránsito colónico con marcadores radioopacos: este estudio estaría indicado en aquellos pacientes cuya evaluación clínica orienta hacia un enlentecimiento del transito colónico. El paciente ingiere una cápsula que contiene 24 marcadores radio-opacos. El día 5 se realiza una radiografía de abdomen y se llega al diagnóstico de tránsito colónico anormal cuando más del $20 \%$ del marcador persiste en el colon o recto. Durante el período de 5 días el paciente debe evitar el uso de laxantes o enemas.

Otra forma de evaluar el tránsito colónico es por medio de un estudio centellográfico. El paciente ingiere cápsulas de liberación prolongada radiomarcadas diseñadas para ser liberadas en el íleon distal y vaciadas en forma de bolo en el colon. Se cuantifica el tránsito colónico durante 24 hs. Si bien es un estudio que se puede realizar en forma rápida, se debe considerar que es un estudio caro y requiere de la disponibilidad de materiales especiales. 10

Evaluación de la disfunción del piso pelviano: la defecografía provee una evaluación dinámica de la defecación y es útil en detectar rectocele, enterocele, intususcepción rectal y descenso perineal patológico.

El estudio consiste en opacificar el recto introduciendo una pasta de sulfato de bario para estimular la defecación. Luego el paciente se sienta en un inodoro radiolucente y realiza una serie de maniobras para evaluar los mecanismos de continencia y medir el descenso de los músculos del piso pelviano. Se realiza además una filmación durante la defecación y posterior a la misma. 9

Manometría ano-rectal: Provee información acerca del tono de reposo del esfínter anal y de la presión durante el esfuerzo. La manometría también incluye la evaluación de la presencia o ausencia del reflejo inhibitorio recto-anal (fundamental para descartar enfermedad de Hirschprung). La presencia de una presión de reposo elevada sugiere anismo. 
Expulsión del balón: se lo utiliza como rastreo de disfunción del piso pelviano (aunque según algunos trabajos es un test insensible). Se introduce en el recto un balón de 50-60 ml. y se infla con el paciente en decúbito lateral izquierdo. Se le indica al paciente expulsar el balón. Si ésta maniobra no puede ser realizada en forma espontanea, se insufla el balón de forma progresiva para facilitar la expulsión.

El rango normal de peso agregado es de 0 a 200 grs. La imposibilidad de expulsar el balón sugiere disfunción del piso pelviano o anismo.

Manejo específico del paciente con desórdenes fisiológicos Constipación por transito colónico lento: el tratamiento inicial consiste en laxantes que aumenten el volumen de las heces, ablandadores de la materia fecal y/o agentes osmóticos. Cuando todo lo anterior falla se usan estimulantes de la motilidad. En los pacientes en los que ha fallado el tratamiento médico agresivo está indicada la cirugía (colectomía o hemi-colectomía). Es importante en estos casos descartar previamente la disfunción del piso pelviano para evitar fallas en la respuesta a la cirugía. Esta última resultaría particularmente útil en los pacientes con inercia colónica. ${ }^{11}$

Disfunción del piso pelviano: La respuesta al tratamiento médico con laxantes ha demostrado ser variable.

Otra opción es el re-entrenamiento del piso pelviano con biofeed-back, observándose una respuesta satisfactoria en el $70 \%$ de los pacientes. Parecería que aquellos pacientes con un descenso perineal inferior a $3,3 \mathrm{~cm}$. tendrían mejor respuesta que aquellos que tienen un descenso mayor.

El re-entrenamiento también resultaría útil en los pacientes en quienes no se encuentra una causa evidente de constipación, y consiste en la modificación de los hábitos, como por ej. hacerse una rutina diaria de evacuación intestinal preferentemente 10 min. luego de la ingesta a fin de aprovechar el reflejo gastrocólico, prestar atención al deseo evacuatorio y no prolongarlo. 12 Muchas veces en pacientes crónicamente constipados los enemas o supositorios pueden ser útiles para estimular el deseo defecatorio

\section{Para llevar al consultorio}

\section{Diagnóstico}

- Podemos decir que hay constipación ante cualquier disminución del ritmo evacuatorio habitual + disconfort Las causas más comunes son bajo aporte dietario de fibras, colon irritable y tránsito colónico lento

- Evaluar siempre uso de medicamentos e ingesta de fibra, frecuencia evacuatoria, sintomas asociados a la defecación, antecedentes de cirugías pelvianas previas, signos de enfermedades sistémicas y un completo examen rectal

- La constipación crónica suele asociarse a un problema funcional y al. uso crónico de laxantes irritativos.

- Los pacientes con constipación idiopática generalmente no presentan otros síntomas aparte de la constipación.

- Si la constipación comienza luego de los 50 años se deben considerar estudios complementarios. La presencia de sangre, pérdida de peso y/o anemia obligan a descartar un cáncer de colon

\section{Manejo}

- Los pacientes sin otros signos de organicidad, pueden seguirse por algunas semanas con un manejo conservador (fibras, actividad física, determinación de sangre oculta en materia fecal y reeducación del hábito evacuatorio) antes de decidir un estudio del colon.

- En el tratamiento juega un rol fundamental la educación, la dieta rica en fibras, la actividad física, suspender toda mediación no esencial que disminuya la motilidad colónica y discontinuar los laxantes irritativos.

- Si se requiere de un laxante, es preferible emplear los de volumen como el psyllium y si el paciente es refractario e insiste en recibir medicación, puede prescribirse lactulosa o sorbitol.

- El restablecimiento del funcionamiento colónico lleva semanas. El paciente debe saberlo para que no recurra nuevamente a los laxantes irritativos.

\section{Dra. María Gabriela Fabbro [ Servicio de Clínica Médica. Hospital Italiano de Buenos Aires ]}

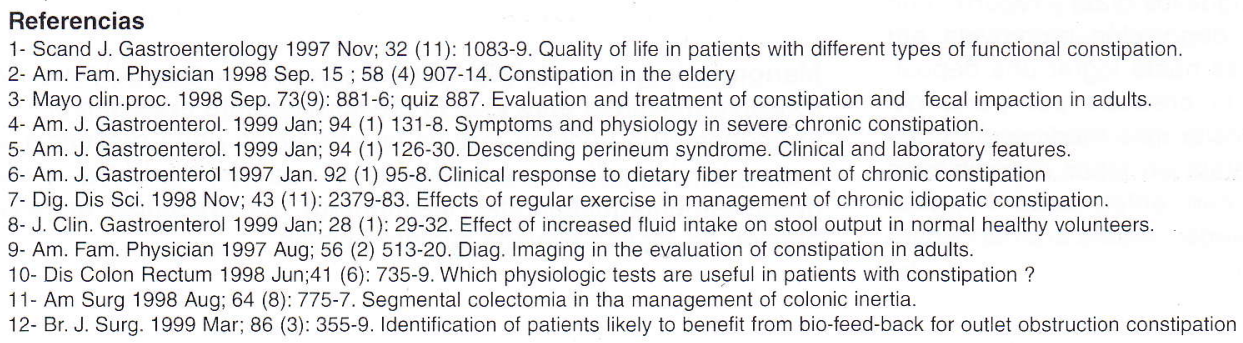

